\title{
EXPERIENCIA DE MANEJO SILVOPASTORAL EN DOS RENOVALES COETANEOS DE Nothofagus antarctica (G.Forst.) Oerst. (Ñire) EN LA REGIÓN DE AYSÉN, CHILE
}

Salinas, Jaime ${ }^{5}$

\section{RESUMEN}

La estructura y dinámica de los bosques de Nothofagus de Sudamérica está asociada a disturbios o alteraciones periódicas, tales como volteos por viento, incendios, movimientos de masas y tectonismo. Los disturbios a pequeña escala, donde solo se produce la caída de árboles individuales o de un grupo de ellos, dan lugar a una dinámica de claros o "gaps". Los bosques de ñire (Nothofagus antarctica) no se alejan de esta dinámica de disturbios, estos ecosistemas durante largo tiempo se asocian a una presión de ganado doméstico, al ataque de insectos por su distribución de transición estepárica y han sido asolados durante décadas por incendios forestales, resultando esto en una perdida estructural y un escaso valor comercial. El tamaño arbustivo o achaparrado de la especie, con troncos retorcidos, no ha permitido que sea utilizada con fines madereros.

Desde el punto de vista botánico, los bosques de ñire se adscriben mayoritariamente a la clase Nothofagetea pumilionis-antarcticae. Se los clasifica en tres morfotipos para ñire en el centrosur de Chile, atendiendo al desarrollo del sistema aéreo vegetativo; arborescente, arbustivo achaparrado y camefítico. Esta plasticidad ecomorfológica le permite a la especie tolerar variadas y rigurosas condiciones físicas, con grandes variaciones térmicas diarias y déficits de saturación atmosférica, y le ha permitido ser la especie que alcanza la mayor amplitud ecológica de los Nothofagus sudamericanos. En Aysén el ñire habita una amplia diversidad de ambientes como fondos de valle, laderas empinadas con suelos someros, ambientes inundables (turberas, mallines), hasta el límite altitudinal de la vegetación, creciendo hasta los $1.200 \mathrm{msnm}$.

Los ecosistemas dominados por ñire en la región de Aysén se extienden latitudinalmente desde los $43^{\circ} 57,9^{\prime}$ LS hasta los $48^{\circ} 29,3^{\prime}$ LS y están presentes en asociación dentro del Tipo Forestal Lenga y clasificados como Subtipo Ñire. Sin embargo, la legislación forestal vigente no los reconoce como tal. El Subtipo Ñire representa regionalmente una superficie de 131.593,4 ha, con presencia en casi todas las comunas a excepción de la comuna de Las Guaitecas. Las mayores poblaciones de ñire se presentan en las Provincias de Coyhaique y Capitán Prat con $61.630,6$ ha y $37.241,3$ ha, respectivamente.

En el presente artículo se presenta una experiencia de investigación de manejo silvopastoral en bosques ñire en la región de Aysén. La iniciativa corresponde a un proyecto de investigación denominado Pautas de Manejo Silvopastoral para Bosques de Nothofagus antarctica en la Región de Aysén, financiado por el Fondo de Investigación del Bosque Nativo (FIBN). El objetivo de dicho proyecto es definir pautas de manejo silvícola para bosques de ñire, que hagan posible la producción silvopastoral sustentable en bosques pertenecientes a pequeños productores forestales de la región.

Palabras claves: Nire, Nothofagus antárctica, Sistemas Silvopastorales, Renovales

\footnotetext{
${ }^{5}$ Ingeniero Forestal, Instituto Forestal, Sede Patagonia, Coyhaique, Chile. jsalinas@infor.cl
} 


\section{SUMMARY}

Structure and dynamics of Nothofagus forests in South America are associated to recurring disturbances and changes such as forest fires, wind damages, mass movements and tectonics. Small scale disturbances, producing individual trees or small stands falls, drive to a dynamic of forest clearings or gaps. Nire (Nothofagus antarctica) forests are not the exception and for a long time have been associated to different pressures because of ranching, forest fires, insetc attacks and other pressuring factors. As a result, most of the remaining forests have lost their structure and commercial value, and right now their shrubby and twisted size has not allowed their use to wood production.

From the botanical point of view, most Nire forests belong to the Nothofagetea pumilionisantarcticae class and according to their aerial vegetative system are classified in to three morphological types; trees, shrubs and camefitic. This ecomorphological plasticity allows the species to tolerate a variety of harsh environmental conditions; such as wide daily thermic variations and atmospheric saturation deficit being the species reaching the wider ecological range within the Southamerican Nothofagus. In the Aysén region Ñire covers a wide environmental diversity; valley bottoms, thin soil slopes and wet areas, reaching also the vegetational altitude limit up to 1,200 masl.

Nire ecosystems in the Aysén region extends between $43^{\circ} 57,9^{\prime} \mathrm{SL}$ and $48^{\circ} 29,3^{\prime} \mathrm{SL}$ and are present in association on the Lenga Forest Type (Nothoifagus pumilio) classified as the Ñire Subtype. However, is not recognized as such a subtype by the current forest legislation. The Nire Subtype covers at the Aysén region 131,593 ha and is present in almost all the communes with the exception of Las Guaitecas commune. Mayor Nire populations are located at the Coyhaique and Capitán Prat provinces, covering 61,630.6 and 37,241.3 ha, respectively.

The present article presents a research experience on silvopastoral systems in Nire forests at the Aysén region. This experience was carried out under de framework of the Silvopastoral Management in Ñire Forests at the Aysén Region research project, supported by the Native Forest Research Fund. Main project objective is the development of silvopastoral management schemes to allow a sustainable production in forests belonging to small owners.

Key words: Ñire, Nothofagus antárctica, Silvopastoral Systems, Second Growth Forests. 


\section{INTRODUCCIÓN}

La Patagonia chilena comprende los territorios de la provincia de Palena en la región de Los Lagos, la región de Aysén y la región de Magallanes.

En la Patagonia norte de Chile, la región de Aysén se ubica entre los $43^{\circ} 38^{\prime} S$ y $49^{\circ} 16^{\prime} S$ (Vergara y Jerez, 2010).

La zona representa una historia reciente de colonización (ILPES 2002, Ortega y Brüning, 2004), que ha transformado grandes extensiones de bosques caducifolios de lenga (Nothofagus pumilio [Poepp. \& Endl.] Krasser) y ñire ( $N$. antarctica [Forster] Oerst.) en explotaciones ganaderas y fragmentos dispersos de estos bosques nativos (Veblen et al., 1996).

Desde un punto de vista ecorregional terrestre, la región de Aysén corresponde a un ecotono entre la zona climática templada septentrional y el dominio austral con marcado acento antártico.

A esta rigurosidad climática, la cual es creciente de Oeste a Este y de Norte a Sur, se agregan las características de un relieve montañoso abrupto y fragmentado por las glaciaciones pleistocénicas (Naruse y Aniya, 1995).

En Aysén los bosques nativos cubren una superficie de 4,3 millones de ha (CONAF, 2012). Los bosques del género Nothofagus son los de mayor relevancia económica en Chile.

Existen 11 taxa de este género en el territorio nacional, correspondientes a 9 especies, un híbrido y una variedad (Ramírez, 1987).

Lenga y ñire son dos de las nueve especies del género con presencia en la zona austral y cubren una superficie de 1.400 .376 ha en la región de Aysén (CONAF, 2012).

En la zona intermedia de Aysén, entre el bosque siempreverde y la estepa patagónica, originalmente dominada por los bosques caducifolios de lenga y ñire, extensas explotaciones ganaderas y fragmentos dispersos de bosque nativo y plantaciones dan forma a los amplios valles ganaderos (Hepp et al., 1988).

El uso ganadero en los bosques en la región, se remonta a la introducción del ganado ovino y bovino durante la colonización en el siglo XIX. En un comienzo, la ganadería ejerció una fuerte presión sobre el bosque, que se mantiene en la actualidad.

En Aysén aún existe controversia en el área total deforestada, la pérdida de superficie de bosque original a partir de 1930 se estima en más del $60 \%$ de la superficie original (Veblen et al., 1996).

En Patagonia Sur (Argentina) se ha desarrollado investigación sobre sistemas silvopastoriles basados en la ganadería bovina y ovina en zonas de bosque de ñire.

En Chile existe escasa información sobre sistemas de producción mixto silvopastoral en bosques nativos. Por lo anterior, en el presente trabajo se examina una experiencia de manejo silvopastoral en dos condiciones de bosques de Nothofagus antarctica realizados en la región de Aysén de Chile. 


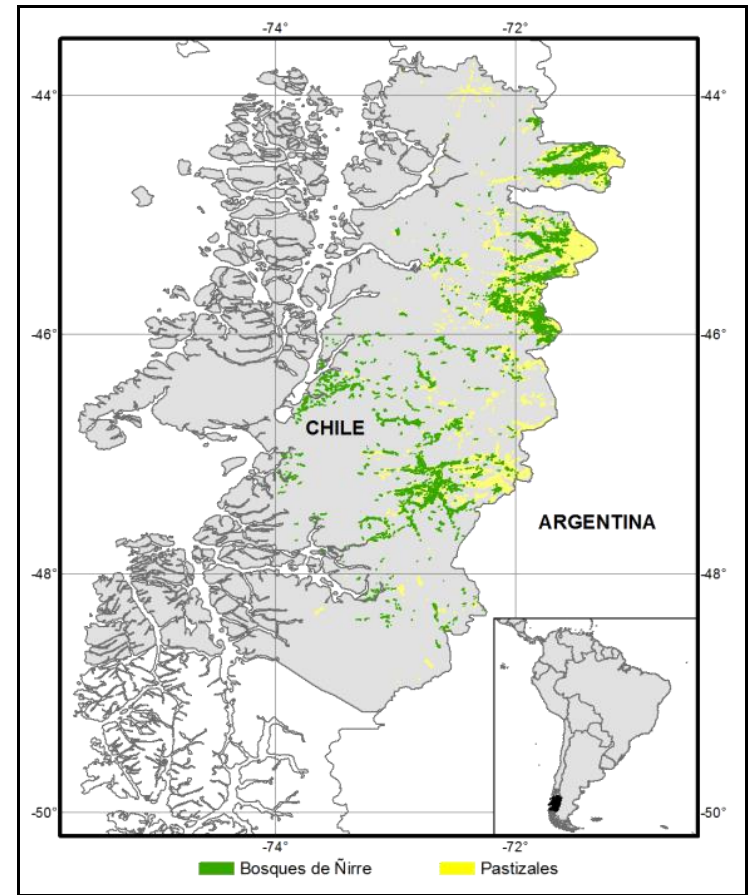

(Fuente: Elaboración propia y CONAF, 2012)

Figura $\mathrm{N}^{\circ} 1$

BOSQUES DE ÑIRE Y ZONAS DE PASTIZALES EN LA REGIÓN DE AYSÉN

Cuadro $\mathrm{N}^{\circ} 1$

SUPERFICIE DE BOSQUES DE ÑIRE, CLASIFICADOS DENTRO DEL TIPO FORESTAL LENGA Y MATORRALES DE ÑIRE, REGIÓN DE AYSÉN

\begin{tabular}{|c|c|c|c|c|}
\hline \multirow{3}{*}{ Provincia } & \multirow{3}{*}{ Comuna } & \multicolumn{2}{|c|}{ Tipo Forestal Lenga } & \multirow{2}{*}{ Matorrales de Ñire } \\
\hline & & Subtipo Ñire & Ñre Especie Dominante & \\
\hline & & & (ha) & \\
\hline \multirow{4}{*}{ Aysén } & Cisnes & 298,6 & 275,2 & $1.084,1$ \\
\hline & Aysén & $15.838,4$ & 13.938 & $4.754,8$ \\
\hline & Guaitecas & - & - & \\
\hline & Subtotal & 16.137 & $14.213,2$ & $5.838,9$ \\
\hline \multirow{3}{*}{ Coyhaique } & Lago Verde & $26.001,5$ & $25.363,4$ & $16.998,2$ \\
\hline & Coyhaique & $35.629,1$ & $35.160,8$ & $7.464,2$ \\
\hline & Subtotal & $61.630,6$ & $60.524,2$ & $24.462,4$ \\
\hline \multirow{3}{*}{$\begin{array}{l}\text { General } \\
\text { Carrera }\end{array}$} & Chile Chico & 9.800 & $8.908,6$ & $13.563,6$ \\
\hline & Río lbáñez & $6.784,5$ & $6.313,9$ & $2.361,2$ \\
\hline & Subtotal & $16.584,5$ & $15.222,5$ & $15.924,8$ \\
\hline \multirow{4}{*}{$\begin{array}{l}\text { Capitán } \\
\text { Prat }\end{array}$} & Cochrane & $29.058,6$ & $25.128,6$ & $38.364,1$ \\
\hline & Tortel & $5.237,1$ & $5.032,2$ & 4.518 \\
\hline & O`Higgins & $2.945,6$ & $2.684,3$ & $4.493,5$ \\
\hline & Subtotal & $37.241,3$ & $32.845,1$ & $47.375,6$ \\
\hline \multicolumn{2}{|l|}{ Total } & $131.593,4$ & 122.805 & $93.601,7$ \\
\hline
\end{tabular}

(Fuente: CONAF, 2012 y Elaboración propia) 


\section{MATERIAL Y MÉTODO}

\section{Área de Estudio}

El presente estudio se llevó cabo en dos ñirantales de la comuna de Coyhaique, que poseen diferencias edafoclimaticas marcadas. El primero corresponde al predio Vista Hermosa ubicado en un sitio en el sector de Balmaceda ( $45^{\circ}$ 52' 22" LS y $71^{\circ} 49^{\prime}$ '08" LW) a 530 msnm, caracterizado por un bosque secundario coetaneo de ñire sobre un mallín (napa freática superficial). El segundo sitio está cercano, en el predio Los Mallines en el sector denominado Galera Chico ( $45^{\circ} 50^{\prime} 25^{\prime \prime}$ LS y $71^{\circ} 50^{\prime} 01^{\prime \prime}$ LW) a $580 \mathrm{msnm}$, donde ocurre un boque coetaneo en transición con la estepa (condición seca). El uso histórico de ambos ñirantales ha sido el de ganadería bovina extensiva desde principios del siglo XX. En ambos casos se compara la situación de un bosque sin intervenir (testigo forestal), con un bosque intervenido (raleo por lo bajo) y la situación pastoril sin bosque (pradera aledaña al bosque, para analisis de la estrata herbacea).

\section{Cuadro $\mathrm{N}^{\circ} 2$ \\ TRATAMIENTOS EVALUADOS EN DOS CONDICIONES DE NIIRANTAL COMUNA COYHAIQUE, CHILE}

\begin{tabular}{|c|l|l|}
\hline Tratamiento & Tratamiento & Descriptor \\
\hline A & Testigo forestal & Bosque de ñire sin intervenir \\
\hline B & Silvopastoral & Bosque de ñire raleado (40\% área basal) \\
\hline C & Pradera natural & Situación pastoril (100\% transmisividad luminica) \\
\hline
\end{tabular}

\section{Componente Forestal}

La caracterización de la estructura forestal fue evaluada a partir de un inventario de 10 parcelas circulares de $100 \mathrm{~m}^{2}$ distribuidas al azar en cada condición de bosque de ñire. Para el caso del tratamiento Silvopastoral la medición se realizó antes y después de la intervención.

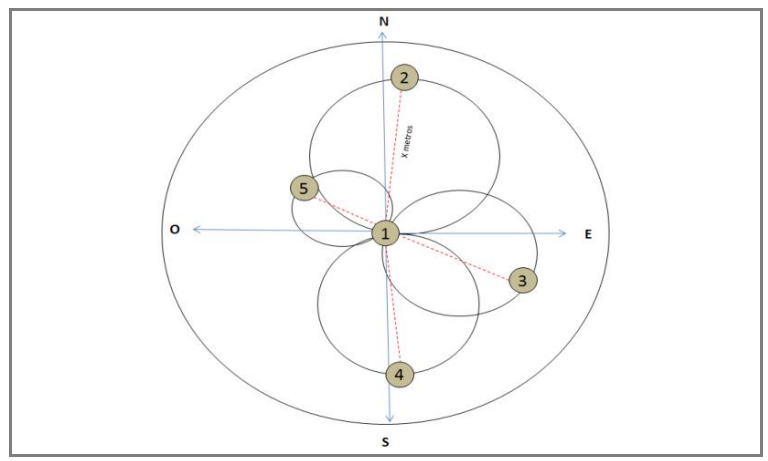

Figura $\mathrm{N}^{\circ} 2$

DIAGRAMA DE MEDICIÓN DEL DISTANCIAMIENTO MEDIO ENTRE ÁRBOLES

En cada parcela se midieron el diámetro a 1,3 m (DAP), la altura total (medida con Vertex), la cobertura de copas (medida con fotografías hemisféricas) y la regeneración existente se evaluará a través de cuatro parcelas de $1 \mathrm{~m}^{2}$, cada una dispuesta en el sentido de los puntos cardinales (N, S, E, O). El distanciamiento medio entre árboles fue determinado a través de la selección de un árbol central y medición de los cuatro árboles más cercanos, orientados en los puntos cardinales (Figura № 2). 
A partir de la información del inventario se estimó el área basal $\left(\mathrm{m}^{2} / \mathrm{ha}\right)$, densidad de la población (arb/ha), el diámetro medio cuadrático $(\mathrm{cm})$ y las existencias $\left(\mathrm{m}^{3} \mathrm{scc} / \mathrm{ha}\right)$, para estimar este parámetro se utilizó la función de volumen propuesta por Cisternas et al. (2015).

$$
\text { Volumen total }\left(m^{3} \mathrm{scc}\right)=0,0000426656{ }^{*} \mathrm{DAP}^{\wedge} 2{ }^{*} \mathrm{HT} \quad \mathrm{R}^{2}=99,9633
$$

La evaluación y monitoreo de la dinámica y estructura forestal a largo plazo se evaluará a partir de un conglomerado, compuesto de 3 parcelas permanentes circulares de $200 \mathrm{~m}^{2}$ (radio= $7,98 \mathrm{~m}$ ) distribuidas sistemáticamente en forma de " $\mathrm{L}$ " invertida. Cada árbol de la parcela fue identificado, numerado y marcado a la altura de 1,3 $\mathrm{m}$ para tener mayor precisión en las mediciones anuales. Para el levantamiento de la información dasométrica se medirá de cada árbol de las parcelas la altura total utilizando Vertex, el DAP con forcípula, y las clases de copa (dominante, codominante, intermedia y suprimida). La variación lumínica (cobertura de copas) y la regeneración natural serán evaluadas usando la misma metodología anteriormente descrita. A partir de mediciones anuales en el mes de mayo, se estimará el área basal, dinámica de densidad (mortandad) y crecimiento en volumen del rodal.

Garantizar la continuidad del estrato arbóreo es un componente central en la implementación de sistemas silvopastorales (Peri et al., 2009), con este fin se abordan distintos ensayos; estudio de germinación de semillas, enraizamiento de estacas, regeneración natural en el boque y regeneración agámica.

\section{Estudios de Germinación}

Se realizaron análisis físicos de semillas de ñire siguiendo la metodología de la norma ISTA (International Seed Testing Association). Para determinar los parámetros de energía germinativa, periodo de energía y capacidad germinativa se usó la metodología del valor máximo de Czabator (1962). Se montaron cuatro repeticiones de 50 semillas para cada tratamiento (Cuadro $N^{\circ} 3$ ) en placas Petri con papel humedecido, para luego ser dispuestas en cámara germinadora, en ausencia de luz, a temperatura constante de $23^{\circ} \mathrm{C}$.

\section{Cuadro $\mathrm{N}^{\circ} 3$}

TRATAMIENTOS PREGERMINATIVOS DE SEMILLAS

\begin{tabular}{|c|l|c|}
\hline Tratamiento & \multicolumn{1}{|c|}{ Descriptor } & $\begin{array}{c}\text { Semillas } \\
\left(\mathbf{N}^{\circ}\right)\end{array}$ \\
\hline E1 & Control sin estratificación & 200 \\
\hline E2 & Estratificación 30 días & 200 \\
\hline E3 & Estratificación 45 días & 200 \\
\hline E4 & Estratificación 60 días & 200 \\
\hline
\end{tabular}

\section{Enraizamiento de Estacas}

Para realizar las pruebas de producción vegetativa se colectó material vegetal procedente de las dos condiciones de sitio del ñirantal (mallín, seco). Este material fue obtenido del último crecimiento anual o bianual de cada árbol seleccionado (Salinas et al., 2011) y evaluado a través de tres repeticiones de 20 estacas a diferentes concentraciones de ácido indolbutírico (T1= 0 ppm, T2 $=3.000$ ppm, T3=100 ppm, T4= 200 ppm, T5= 500 ppm, T6=1.000 ppm, T7=2.000 ppm).

\section{Regeneración Natural}

La evaluación de la regeneración natural existente en cada condición de sitio fue evaluada considerando presencia y ausencia de ganado. En cada parcela forestal se evaluó la 
regeneración natural con presencia de pastoreo, a través de cuatro parcelas de $1 \mathrm{~m}^{2}$, distanciadas $5 \mathrm{~m}$ desde el árbol central y orientada en el sentido de los puntos cardinales (N, S, E, O).

El total de parcelas a evaluar en cada tratamiento (testigo forestal, silvopastoreo) fue de 12 parcelas por sitio. En paralelo se instalaron tres jaulas metálicas de $1 \mathrm{~m}^{2}$, con ello se evaluó la instalación de plántulas de regeneración natural sin presencia de ganado (aislando la herbivoría).

Ambas evaluaciones se realizaron al inicio (diciembre) y final (abril) de cada período de crecimiento (Bahamonde et al., 2011).

\section{Regeneración Agámica}

El ñire presenta alta capacidad de reproducción vegetativa, reproduciéndose por raíz, tocón y esquejes, gracias a estas características la silvicultura de monte bajo se debe considerar relevante para asegurar el bosque en sistemas mixtos silvopastorales.

En este contexto, en el bosque manejado (tratamiento silvopastoral) de ambos sitios, se protegieron 18 tocones utilizando dos tipos de protectores individuales; el primero (T1) construido con malla gallinera (aislando vacuno y liebre), el segundo (T2) de alambre liso y púas (aislando vacuno), y (T3) sin protector.

En cada repetición se evaluaron parámetros del tocón (DAT: diámetro altura tocón, Htoc: altura tocón, $\mathrm{Nb}$ : número de brotes, Lb: largo de brotes y $\mathrm{Nm}$ : número de muñones) y porcentaje de ramoneo para cada tratamiento.

\section{Componente Herbáceo y Suelo}

Previo al inicio del experimento se tomó una muestra de suelo, para cada tratamiento en los dos sitios experimentales, con el propósito de evaluar la composición química inicial (micro y macroelementos) de cada sector.

Para ello se realizó un muestreó en cada sitio mediante un barreno $(10 \mathrm{~cm}$ profundidad). En cada caso se tomaron 20 submuestras, las que luego fueron mezcladas para formar una muestra compuesta, la que se destinó a análisis en laboratorios de INIA.

En cada unidad experimental (testigo forestal, silvopastoral y pradera natural) se instalaron cuatro jaulas de exclusión $\left(0,5 \mathrm{~m}^{2}\right)$, las cuales fueron distribuidas al azar, en sitios donde existiera cierta cubierta herbácea, aunque esta fuera incipiente.

Cuando la estrata herbácea al interior de la jaula alcanzaba una altura factible de ser muestreada (aproximadamente $15-20 \mathrm{~cm}$ ), se procedía a su cosecha, removiendo la jaula y colocando un marco de la misma medida $\left(0,5 \mathrm{~m}^{2}\right)$ para cortar con una maquina eléctrica todo el material vegetal presente.

Luego se procede a reubicar la jaula en un lugar con similares características al que estaba. Este procedimiento se repitió en cada jaula.

El material colectado se pesa posteriormente en verde, para determinar la producción total de materia fresca.

La muestra se divide en dos fracciones; una para determinar el contenido de materia seca (MS) y otra para composición botánica (separación de especies o grupos de especies constituyentes).

Finalmente, para determinar materia seca, la muestra ingresa a una estufa de secado a $60^{\circ} \mathrm{C}$ por aproximadamente 48 horas hasta peso constante. 


\section{RESULTADOS}

\section{Evaluación Forestal}

El estudio se realizó en dos renovales de ñire, ubicado a $45 \mathrm{~km}$ al sudeste de la ciudad de Coyhaique. La evaluación forestal inicial permitió describir la estructura de los ñirantales en estudio. En el Cuadro № 4 se presentan los parámetros dasométricos de los tratamientos testigo forestal y silvopastoral en las dos condiciones de sitio antes de la intervención.

\section{Cuadro $\mathrm{N}^{\circ} 4$}

VARIABLES DE ESTRUCTURA FORESTAL EN DOS CONDICIONES DE BOSQUES DE ÑIRE PROCEDENTES DE PARCELAS PERMANENTES ANTES DE LA INTERVENCIÓN

\begin{tabular}{|l|l|r|r|r|r|r|}
\hline Sitio & Tratamiento & \multicolumn{1}{|c|}{$\begin{array}{c}\text { AB } \\
\left(\mathbf{m}^{2} / \mathbf{h a}\right)\end{array}$} & \multicolumn{1}{c|}{$\begin{array}{c}\text { HD } \\
(\mathbf{m})\end{array}$} & \multicolumn{1}{c|}{$\begin{array}{c}\text { DMC } \\
(\mathbf{c m})\end{array}$} & \multicolumn{1}{|c|}{$\begin{array}{c}\mathbf{N} \\
(\mathbf{a r b} / \mathbf{h a})\end{array}$} & \multicolumn{1}{|c|}{$\begin{array}{c}\text { VOL } \\
\left(\mathbf{m}^{3} / \mathbf{h a}\right)\end{array}$} \\
\hline \multirow{2}{*}{ Mallín } & Testigo forestal & 34,74 & 8,1 & 8,3 & 6.370 & 78,50 \\
& Silvopastoral & 45,31 & 9,3 & 10,0 & 5.737 & 132,94 \\
\hline \multirow{2}{*}{ Seco } & Testigo forestal & 42,67 & 9,0 & 9,4 & 6.120 & 117,84 \\
& Silvopastoral & 38,99 & 10,2 & 11,74 & 3.600 & 141,07 \\
\hline
\end{tabular}

$\mathrm{AB} \quad=$ área basal

$\mathrm{HD} \quad=$ altura dominante

$\mathrm{DMC} \quad=$ diámetro medio cuadrático

$\mathrm{N} \quad$ = densidad de individuos

$\mathrm{V} \quad=$ volumen total

En ambas condiciones de sitio, el bosque presenta una estructura regular de origen monte medio, donde domina la presencia de cepas de 3 a 8 pies y en menor proporción individuos de monte alto. La estrategia de intervención correspondió a un raleo de selección positiva, la que permitió un incremento del DMC sin alterar el estado de desarrollo de Latizal Bajo. La densidad del bosque original fue de $6.370 \mathrm{arb} / \mathrm{ha}$ para el sector de mallín y $6.120 \mathrm{arb} / \mathrm{ha}$ para la condición seca, mientras que los tratamientos silvopastorales presentaron densidades de 5.737 y 3.600 arb/ha para la condición de mallín y seca, respectivamente, las que fueron reducidas en un 55\% (mallín) y 59\% (seca) (Figura № 3).

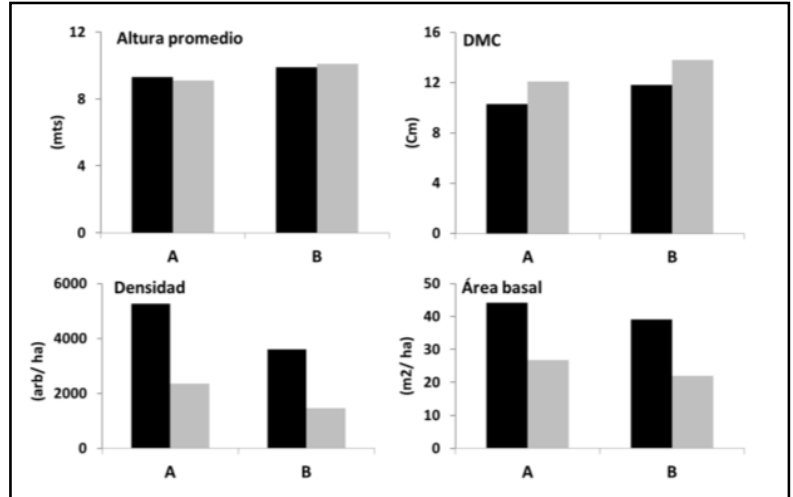

El color negro de las barras representa dosel completo y el color gris dosel intervenido

Figura 3

IMPACTO DE LA APERTURA DEL DOSEL SOBRE VARIABLES FORESTALES EN DOS CONDICIONES DE BOSQUES 


\section{- Luminosidad}

Las variables fotométricas y radiométricas se evaluaron a través de fotografías hemisféricas (lente SUNEX fisheye, campo visual 185). Estas fotografías fueron tomadas evitando la influencia directa del sol, bajo un cielo cubierto de nubes, temprano en la mañana o durante la tarde luego de la caída del sol (Roxburgh \& Kelly, 1995).

El análisis de 10 fotografías antes y otras 10 fotografías después del raleo (Figura № 4), permitió cuantificar el cambio en el régimen lumínico a nivel de rodal.
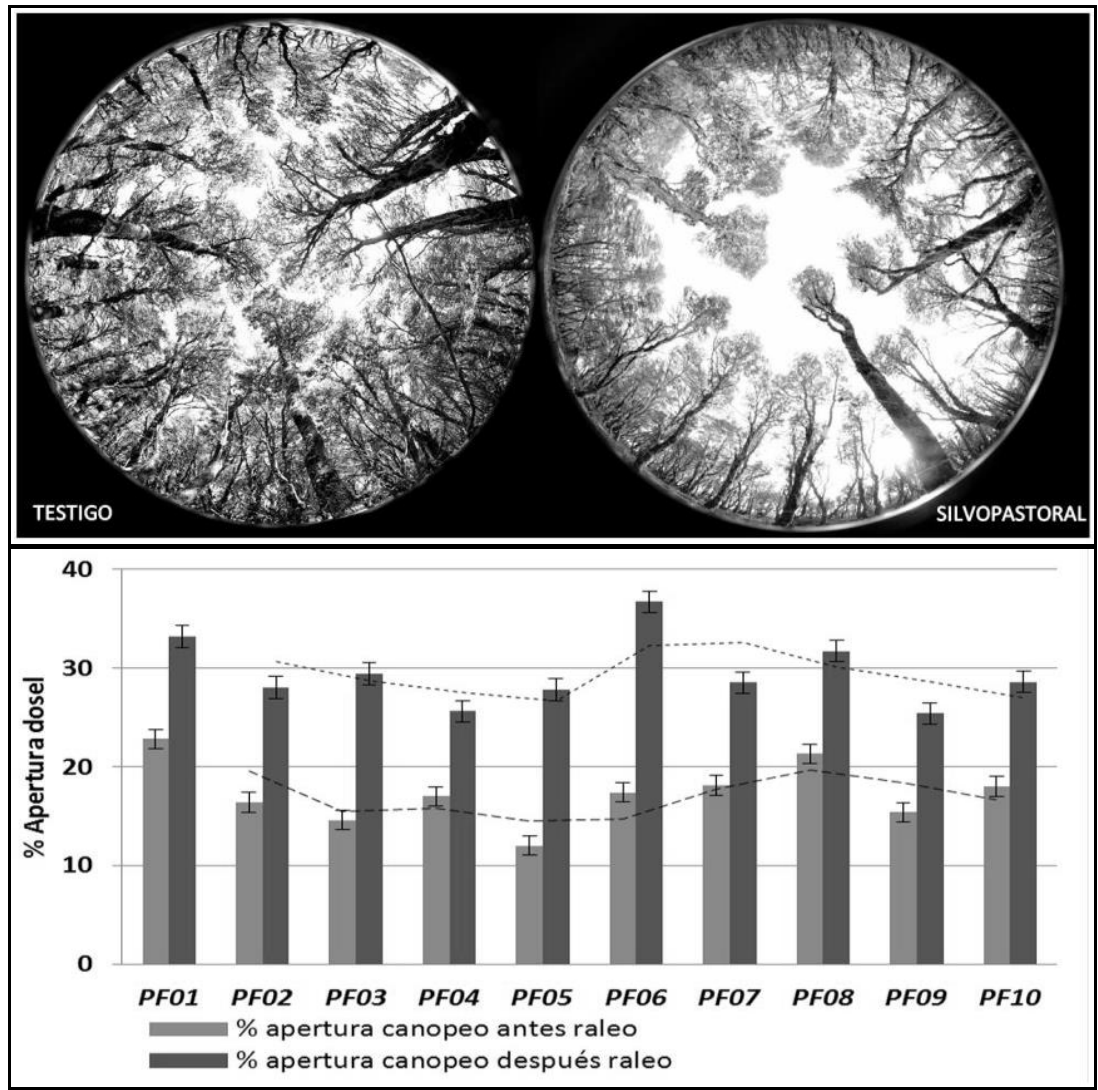

Figura $\mathrm{N}^{\circ} 4$

IMPACTO DE UN RALEO SILVOPASTORAL EN LA APERTURA DEL CANOPEO DE UN ÑIRANTAL CERCANO A LA ESTEPA (CONDICIÓN SECA) UTILIZANDO FOTOGRAFÍAS HEMISFÉRICAS

Para estimar la cobertura del dosel en cada condición boscosa se evaluaron fotografías hemisféricas en tres parcelas permanentes en orientaciones norte-sur y este-oeste. La cobertura boscosa antes de la intervención fue de $72,5 \%$ en el ñirantal en condición seca y $71,4 \%$ en condición de mallín (Cuadro № 5). respectivamente.

Posterior al raleo los parámetros cambiaron moderadamente a $46,2 \%$ y $46,9 \%$ 


\section{PARÁMETROS DE COBERTURA DEL DOSEL EN DIFERENTES CONDICIONES DE ÑIRANTAL EVALUADO A TRAVES DE FOTOGRAFÍAS HEMISFÉRICAS}

\begin{tabular}{|l|l|c|c|c|c|c|c|c|}
\hline \multirow{3}{*}{ Sitio } & \multirow{3}{*}{ Tratamiento } & \multicolumn{5}{|c|}{$\begin{array}{c}\text { Cobertura del Dosel } \\
\text { (\%) }\end{array}$} & \multirow{2}{*}{$\begin{array}{c}\text { Media } \\
\text { ( } \pm \text { DE) }\end{array}$} \\
\cline { 3 - 8 } & & \multicolumn{3}{|c|}{ N - S } & \multicolumn{3}{c|}{ E - O } \\
\cline { 3 - 8 } & & P1 & P2 & P3 & P1 & P2 & P3 & \\
\hline \multirow{2}{*}{ Mallín } & Testigo forestal & 69,49 & 70,24 & 69,30 & 71,30 & 74,22 & 74,13 & $71,4 \pm 2,2$ \\
& Silvopastoral & 46,60 & 47,71 & 48,57 & 45,75 & 46,45 & 46,40 & $46,9 \pm 1,0$ \\
\hline \multirow{2}{*}{ Seco } & Testigo forestal & 72,76 & 72,55 & 71,10 & 74,45 & 73,25 & 70,59 & $72,5 \pm 1,4$ \\
& Silvopastoral & 49,50 & 45,04 & 44,90 & 46,20 & 44,90 & 46,61 & $46,2 \pm 1,8$ \\
\hline
\end{tabular}

$\mathrm{DE}=$ Desviación estándar.

\section{Parámetros Reproducción Sexual y Asexual}

Entre los parámetros físicos de semillas de ñire se determinó el peso de semillas, que corresponde a una variable establecida por las normas internacionales ISTA y consiste en el peso de 1.000 semillas a fin de conocer el tamaño y viabilidad de las especies. Mientras menor sea el peso de una semilla, más pequeño será su tamaño o más baja su viabilidad (Escobar, 2012).

\section{Cuadro $\mathrm{N}^{\circ} 6$ \\ PARÁMETROS FÍSICOS DE SEMILLAS EN DOS CONDICIONES DE SITIO}

\begin{tabular}{|c|c|c|c|c|c|c|}
\hline \multirow[t]{2}{*}{ Sitio } & \multirow[b]{2}{*}{ (año) } & \multirow{2}{*}{$\begin{array}{c}\text { Pureza } \\
\text { (\%) }\end{array}$} & \multirow{2}{*}{$\begin{array}{l}\text { Semillas } \\
\left(\mathbf{N}^{\circ} / \mathbf{k g}\right)\end{array}$} & \multirow{2}{*}{$\begin{array}{c}\text { Peso Semillas } \\
(\mathbf{g})^{\star}\end{array}$} & \multicolumn{2}{|c|}{ Tamaño Semillas } \\
\hline & & & & & $\begin{array}{l}\text { Largo } \\
(\mathrm{mm})\end{array}$ & $\begin{array}{c}\text { Ancho } \\
\text { (mm) }\end{array}$ \\
\hline Mallín & $\begin{array}{l}(2014) \\
(2015)\end{array}$ & $\begin{array}{l}95,8 \\
98,8\end{array}$ & $\begin{array}{l}784.450 \\
972.000\end{array}$ & $\begin{array}{l}1,27 \\
1,45\end{array}$ & $\begin{array}{l}3,10 \pm 0,40 \\
2,87 \pm 0,30\end{array}$ & $\begin{array}{l}3,30 \pm 0,50 \\
2,91 \pm 0,43\end{array}$ \\
\hline Seco & (2015) & 98,6 & 718.000 & 1,63 & $2,44 \pm 0,20$ & $2,41 \pm 0,35$ \\
\hline
\end{tabular}

${ }^{*} \mathrm{~N}=1.000$ semillas.

El peso de semillas de ñire procedente de un ñirantal en condición de mallín vario entre 1,27 y $1,45 \mathrm{~g}$ y el número de semillas por kilogramo entre el rango de 784.450 y $972.000 \mathrm{sem} / \mathrm{kg}$.

El ñirantal en condición seca en tanto presento un mayor peso de semillas de 1,63 g y menor cantidad de semillas por kilogramo $(718.000 \mathrm{sem} / \mathrm{kg})$.

Valores similares fueron entregados por Bahamonde et al. (2013), quienes evaluaron el peso de 1.000 semillas de ñire en diferentes clases de sitio en la Patagonia argentina, encontrando valores entre los rangos 1,1 y $1,6 \mathrm{~g}$.

Los parámetros germinativos para ñire presentan baja capacidad de germinación, alcanzando un máximo de $28 \%$ (estratificación de 45 días).

Similares resultados fueron presentados por Premoli (1991), quien observó que las respuestas a la germinación fueron muy bajas, por diversos factores, entre ellos; herbivoría por insectos, en particular del Orden Lepidoptera (Gentili y Gentili, 1988), generación de tumores por ataque de bacterias, hongos y/o virus (Braun, 1969), o desarrollo partenocárpico de las semillas por tratarse probablemente de un año de escasa floración (Poole, 1950). 


\section{Cuadro $\mathrm{N}^{\circ} 7$}

PARÁMETROS DE GERMINACIÓN DE SEMILLAS

PROCEDENTES DE LA CONDICIÓN DE MALLÍN

\begin{tabular}{|c|c|c|c|c|c|c|c|}
\hline \multirow[t]{2}{*}{ Sitio } & \multirow[t]{2}{*}{$\begin{array}{c}\text { Tratamiento } \\
\text { Pregerminativo }\end{array}$} & \multicolumn{2}{|c|}{$\begin{array}{c}\text { Capacidad } \\
\text { Germinativa } \\
(\%)\end{array}$} & \multicolumn{2}{|c|}{$\begin{array}{c}\text { Energía } \\
\text { Germinativa } \\
(\%)\end{array}$} & \multicolumn{2}{|c|}{$\begin{array}{l}\text { Periodo de } \\
\text { Energía } \\
\text { (Días) }\end{array}$} \\
\hline & & 2014 & 2015 & 2014 & 2015 & 2014 & 2015 \\
\hline \multirow{4}{*}{$\begin{array}{l}\text { Mallín } \\
\left(45^{\circ} 52^{\prime} \text { LS }\right. \\
71^{\circ} 49^{\prime} \text { LW) }\end{array}$} & E1 & 14,5 & 2,7 & 12,5 & 2,7 & 12 & 18 \\
\hline & E2 & 16,0 & 12,0 & 14,0 & 9,3 & 13 & 8 \\
\hline & E3 & 28,0 & - & 21,5 & - & 12 & - \\
\hline & E4 & - & 2,0 & - & 1,3 & - & 7 \\
\hline \multirow{4}{*}{$\begin{array}{l}\text { Seco } \\
\left(45^{\circ} 50^{\prime} \text { LS }\right. \\
\left.71^{\circ} 50^{\prime} \text { LW }\right)\end{array}$} & E1 & - & 16,7 & - & 13,3 & - & 11 \\
\hline & E2 & - & 19,3 & - & 14,7 & - & 8 \\
\hline & E3 & - & - & - & - & - & - \\
\hline & E4 & - & 6,7 & - & 4,7 & - & 8 \\
\hline
\end{tabular}

La evaluación de la reproducción vegetativa de material procedente de la condición de mallín, presentó los niveles más aceptables de formación de raíces, bajo una concentración de 500 ppm (T5), obteniendo 53\% de enraizamiento a los 60 días en la cama caliente.

El tratamiento sin aplicación de enraizante (T1) presentó nula formación de raíces, confirmando lo presentando por (Salinas et al., 2011; 2014). El material vegetal colectado del ñirantal en condición seca, presentó valores de 7\% de enraizamiento (T4 y T6 a los 60 días), muy por debajo en comparación con el de mallín.

Otra opción para dar continuidad al bosque en sistemas silvopastorales, es a través del estudio de protección de tocones, al menos hasta los $2 \mathrm{~m}$ de altura. En el Cuadro № 8 se muestra la información de la primera temporada de evaluación de tocones protegidos en dos condiciones de bosques de ñire.

Cuadro $\mathrm{N}^{\circ} 8$

EVALUACIÓN DE LOS PARÁMETROS DE TOCÓN Y PORCENTAJE DE RAMONEO DE BROTES DE ÑIRE EN BASE A DOS TIPOS DE PROTECTORES INDIVIDUALES

\begin{tabular}{|c|c|c|c|c|c|c|c|c|c|c|c|c|}
\hline \multirow[b]{2}{*}{$T$} & \multicolumn{6}{|c|}{ Sitio Seco } & \multicolumn{6}{|c|}{ Sitio Mallín } \\
\hline & $\begin{array}{l}\text { Dat } \\
(\mathrm{cm})\end{array}$ & $\begin{array}{l}\text { Htoc } \\
\text { (cm) }\end{array}$ & $\begin{array}{c}\text { Brotes } \\
\left(\mathrm{N}^{\circ}\right)\end{array}$ & $\begin{array}{c}\text { Muñón } \\
\left(\mathbf{N}^{\circ}\right)\end{array}$ & $\begin{array}{c}\text { Largo } \\
\text { Brotes } \\
(\mathrm{cm})\end{array}$ & $\begin{array}{l}\text { R } \\
\text { (\%) }\end{array}$ & $\begin{array}{l}\text { Dat } \\
(\mathrm{cm})\end{array}$ & $\begin{array}{l}\text { Htoc } \\
\text { (cm) }\end{array}$ & $\begin{array}{c}\text { Brotes } \\
\left(\mathrm{N}^{\circ}\right)\end{array}$ & $\begin{array}{c}\text { Muñón } \\
\text { (No) }\end{array}$ & $\begin{array}{c}\text { Largo } \\
\text { Brotes } \\
(\mathrm{cm})\end{array}$ & $\begin{array}{c}\mathbf{R} \\
\text { (\%) }\end{array}$ \\
\hline T1 & 14,22 & 40,92 & 57,89 & 9,11 & 17,21 & 0,0 & 11,91 & 41,22 & 67,74 & 6,56 & 32,16 & 4,0 \\
\hline T2 & 16,24 & 44,33 & 70,63 & 8,50 & 10,29 & 70,0 & 13,59 & 41,78 & 52,67 & 7,89 & 12,65 & 58,3 \\
\hline T3 & 14,94 & 42,28 & 61,00 & 9,14 & 10,14 & 90,0 & 13,00 & 43,80 & 55,40 & 9,20 & 4,90 & 74.4 \\
\hline
\end{tabular}

\footnotetext{
T1 = Protector malla (aislando presión de vacuno - liebre)

T2 = Protector alambre (aislando presión de vacuno)

T3 = Sin protector (libre a ramoneo)

Dat = Diámetro altura tocón

Htoc = Altura tocón

$\mathrm{R}=$ Ramoneo
} 


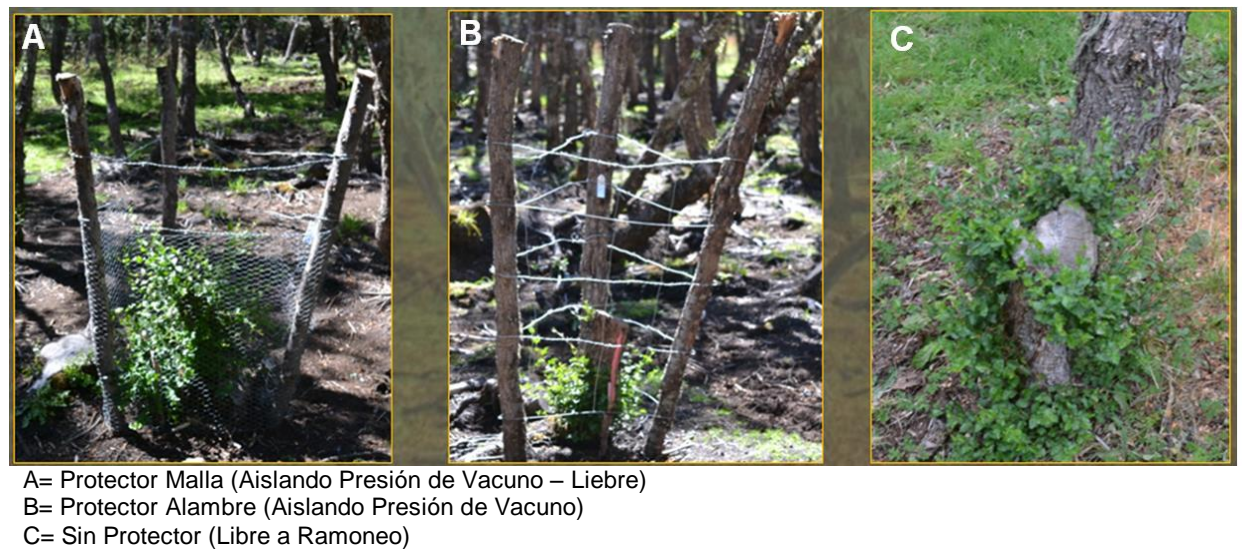

$\mathrm{C}=\mathrm{Sin}$ Protector (Libre a Ramoneo)

\section{Figura $\mathrm{N}^{\circ} 5$ \\ PROTECTORES INDIVIDUALES DE TOCÓN}

\section{CONCLUSIONES}

Desde el punto de vista dasonómico, los bosques estudiados presentan mayoritariamente una forma de masa de monte medio (compuesta por árboles cuyo origen es un brote de cepa o de raíz y regeneración natural). Si bien no se presentaron rastros de acción antrópica, bosques contiguos muestran vestigios de fuego y de aprovechamiento de leña.

La aplicación de raleos en ambos renovales, con una intensidad cercana al $50 \%$ de la densidad, origina una mayor calidad del rodal remanente y un aumento del diámetro medio. Del mismo modo, la estabilidad de la masa remanente del bosque frente al viento y nieve no fue perjudicada producto de la intervención. En paralelo, ocurre una activación pratense producto de mayor luminosidad, aumentando la productividad de la pradera.

La coberturas del dosel en las diferentes condiciones de bosque presentaron diferencias cercanas a un $35 \%$ entre la luminosidad del bosque intervenido (tratamiento silvopastoral) con la del bosque original (testigo). Esta diferenciación fue similar en ambas condiciones de sitio.

Los resultados de germinación obtenidos en laboratorio con una procedencia de semillas de la zona de Coyhaique confirman la baja capacidad germinativa de las semillas de la especie y entregan como mejor resultado el originado con el tratamiento estratificación fría por 45 días, con una germinación acumulada a los 30 días de $28 \%$. El resultado de este tratamiento prácticamente duplica a los obtenidos con el testigo (sin estratificación) y con la estratificación fría por 30 días, que llegaron a $14,5 \%$ y $16 \%$ de germinación, respectivamente, confirmando el efecto positivo de la estratificación fría sobre la capacidad germinativa de ñire. Este resultado indica que la producción de plantas por semilla de ñire es posible, pero con un alto insumo de semillas, y las diferencias mostradas por los tratamientos de estratificación hacen recomendable continuar esta línea de investigación incorporando nuevos períodos de estratificación.

Una alternativa para especies con semillas de baja capacidad germinativa, aunque de mayor costo, es la propagación vegetativa o asexual. En este sentido nuevos estudios debieran incorporar, por ejemplo, material rejuvenecido de rebrotes de cepa, diferentes procedencias del material, variantes de temperatura en cama caliente e invernadero, una época más propicia como la primavera cuando el material vegetal no está en receso invernal y tal vez AIB en mayores concentraciones.

Es necesario considerar criterios o indicadores de clasificación específicos en los 
estudios vinculados a la estructura de los bosques de ñire (como la edad y la calidad de sitio aún no descrita para la especie en la región). No es posible en la descripción de estas masas boscosas, compararlas a otras masas de Nothofagus, que son totalmente disímiles.

\section{RECONOCIMIENTOS}

Se agradece al Fondo de Investigación del Bosque Nativo (FIBN) por el financiamiento del proyecto 022/2013 Pautas de Manejo Silvopastoral de Bosques de Ñire en la Región de Aysén.

Se agradece igualmente al técnico de INFOR Bernardo Acuña y a INIA Tamel Aike por su importante ayuda en el proyecto.

\section{REFERENCIAS}

Bahamonde, H.; Peri, P.; Monelos, L. y Pastur, G., 2011. Aspectos ecológicos de la regeneración por semillas en bosques nativos de Nothofagus antarctica en Patagonia Sur, Argentina. Bosque 32(1): 20-29.

Bahamonde, H.; Peri, P.; Monelos, L. y Pastur, G., 2013. Regeneración por semillas en bosques nativos de Nothofagus antarctica bajo uso silvopastoril en Patagonia Sur, Argentina. Bosque (Valdivia), Vol.34, № 1, p.89101. ISSN 0717-9200.

Braun, A.C., 1969. Abnormal Growth in Plants. En: Plant Physiology, a Treatise, vol. VB, F.C. STEWARD (Ed.). Academic Press, New York: $379 \neg 420$.

Cisternas, J.; Pinto, R. y Cossio, A., 2015. Raleo en bosque de ñire para un aprovechamiento multipropósito. VIII Congreso Internacional de Sistemas Agroforestales. Puerto Iguazú, Misiones, Argentina. 8 pág.

CONAF, 2012. Catastro Vegetacional del Bosque Nativo. Actualización de la XI región de Aysén. Coyhaique, Chile.

Czabator, F. P., 1962. Germination value: An index combining speed and completeness of Pine seed germination. Forest Science 8 (4): $386-396$.

Escobar, R., 2012. Semillas. En: Producción de Plantas en Viveros. Consejo Federal de Inversiones - CIEFAP. 1ra Edición. Buenos Aires. 190 pag.

Gentili, M. y Gentili, P., 1988. Lista comentada de los insectos asociados a las especies sudamericanas del género Nothofagus. Monografías de la Academia Nacional de Cs. Exactas, Físicas y Naturales. Simposio sobre Nothofagus. Bs. As. № 4: 85-105.

Hepp, C.; Thiermann, H. y Ramírez, C., 1988. Praderas en la zona austral XI región (Aysén). In: Ruiz Núñez, I. (Ed.), Praderas para Chile. Instituto de Investigaciones Agropecuarias, Santiago, Chile.

Naruse, R. y Aniya, M., 1995. Synopsis of glacier research in Patagonia. Bulletin of Glaciers Research 13:1-10.

Ortega, H. y Brüning, A., 2004. Aysén. Panorama histórico y cultural de la XI región. Ediciones LOM, Chile. Disponible en http://www.aisenpanorama.cl/libro.htm (07/09/2015).

Peri, P.; Hansen, N.; Rusch, V.; Tejera, L.; Monelos, L.; Fertig, M.; Bahamonde, H. y Sarasola, M., 2009. Pautas de manejo de sistemas silvopastoriles en bosques nativos de Nothofagus antarctica (ñire) en Patagonia. In Actas del Primer Congreso Nacional de Sistemas Silvopastoriles, Posadas, Misiones, Argentina mayo de 2009. Actas. p. 151-155.

Poole, A., 1950. Studies of the New Zealand Nothofagus species. 2. Nut and cupule development. Trans. Roy. Soc. N.Z. 78:502-508.

Premoli, A., 1991. Morfología y capacidad germinativa en poblaciones de Nothofagus antarctica (Forster) Oerst del noroeste andino patagónico. Bosque 12(2): 53-59.

Ramírez, C., 1987. El Género Nothofagus y su Importancia en Chile. Bosque 8(2): 71-76.

Roxburgh, J. R and Kelly, D., 1995. Uses and limitations of hemispherical photography for estimating forest light environments. NZ J Ecol 19:213-217. 
Salinas, J.; Acuña, B.; Ovando, V. y Díaz, E., 2011. Estándares de producción vegetativa en plantas de Nothofagus antarctica (G. Forst.) Oerst. en la región de Aysén. MINAGRI-INFOR. 11p.

Salinas, J.; Acuña, B.; Koch, L. y Uribe, A., 2014. Producción de árboles nativos con fines de recuperación de bosques y áreas degradadas en la región de Aysén. Propagación sexual y asexual de Nothofagus antarctica (Forster) Oerst. Ñire. MINAGRI - INFOR. 53 p.

Veblen, T. T.; Kitzberger, B. y Rebertus, A., 1996. Perturbaciones y dinámica de regeneración en bosques andinos del Sur de Chile y Argentina. In: Armesto, J.J., Villagrán, C., Arroyo, M.K. (Eds.), Ecología de los bosques nativos de Chile. Editorial Universitaria, Santiago de Chile, pp. 169-197.

Vergara, Olivia, y Jerez, Viviane, 2010. Insectos e infestaciones asociadas al follaje de Nothofagus antarctica (Forst) Oerst (Nothofagaceae) en la cuenca del río Baker, región de Aysén, Chile. Gayana (Concepción), 74(2), 83-93. 